\title{
Evaluation Scheme for a Positron Emission Tomography System used in Monitoring of Radiation Therapy
}

\author{
Georgy Shakirin ${ }^{1}$, Fine Fiedler ${ }^{1}$, Wolfgang Enghardt ${ }^{1,2}$ \\ ${ }^{1}$ Institute of Radiation Physics, Forschungszentrum Dresden-Rossendorf, Dresden \\ ${ }^{2}$ OncoRay, Technische Universität Dresden \\ g.shakirin@fzd.de
}

\begin{abstract}
Positron emission tomography (PET) is used for independent monitoring of dose delivery in ion therapy. An in-beam PET scanner registers the annihilation $\gamma$-rays following the decay of $\beta^{+}$-radioactive nuclei produced via nuclear reactions between the ions of the therapeutic beam and the irradiated tissue. From a comparison of the reconstructed activity distributions with those predicted from the treatment plan, deviations between the prescribed and the applied dose can be detected. In-beam PET, therefore, allows to verify the physical beam model of the treatment planning, to detect patient dislocations and density changes in the irradiated tissue. Issues related to the image quality and evaluation of a whole PET imaging system are discussed in this paper.
\end{abstract}

\section{Introduction}

An in-beam PET scanner is used to measure $\beta^{+}$-activity distributions corresponding to the applied dose during therapeutic irradiation. The reconstructed $\beta^{+}$-activity image allows to evaluate the conformity and accuracy of the performed treatment. The main task of in-beam PET is to detect and interpret deviations from the prescribed and actually applied dose. The applied dose is characterized by the measured $\beta^{+}$-activity distribution. Representing different physical processes, dose and $\beta^{+}$-activity distribution cannot be compared directly. Therefore, an expected $\beta^{+}$-activity distribution based on the prescribed dose is simulated [1]. The quality assurance of the irradiation is then performed by a comparison of the expected (corresponding to the prescribed dose) and the measured (corresponding to the actually applied dose) $\beta^{+}$-activity distributions.

The in-beam PET scanner BASTEI (Fig. 1) is installed at the heavy ion therapy facility at the Gesellschaft für Schwerionenforschung (GSI), Darmstadt, Germany [1,2]. Since 1997 irradiation of more than 400 patients have been monitored there by means of in-beam PET. A dedicated, limited angle configuration of the in-beam PET scanner (Fig. 1), a very low counting statistics, and requirement of fast (ideally, real time) availability of in-beam PET images for analysis make the imaging process a challenging task. Based on more than 10 year experience of the clinical exploitation of the in-beam PET technology 
specific quality criteria and an evaluation workflow for an in-beam PET imaging system have been developed.

\section{Materials and methods}

\subsection{Quality criteria for in-beam PET}

Three criteria are used for evaluation of imaging capabilities of an in-beam PET system: root mean square error (RMSE), contrast recovery value for NEMA-like phantom, and range modification trials [3]. RMSE (1) shows the average image noise and is not sensitive to particular image details.

$$
R M S E=\sqrt{\sum_{j=1}^{N}\left(x_{j}-x_{j}^{*}\right)^{2} / N},
$$

where $N$ is a number of elements (pixels for 2D or voxels for 3D) of the image, $x_{j}$ is the reference value of activity, and $x_{j}^{*}$ is the reconstructed activity value at element $j$. NEMA-like phantom (Fig. 2) specially adapted for counting statistics and size of irradiated volume of in-beam PET is used for the contrast recovery calculation (2):

$$
L C R H=\frac{M H-M B}{3 M B} \cdot 100 \% ; \quad L C R C=\frac{M B-M C}{M B} \cdot 100 \%,
$$

where $L C R H$ is local contrast recovery ratio for the high activity areas (four hot spheres), $L C R C$ is local contrast recovery ratio for the areas of no activity (two cold spheres and the central cylinder). $M H, M B$, and $M C$ are median values for activity inside hot spheres, background, and cold areas, respectively. The median value for the background is calculated locally near each cold or hot

Fig. 1. In-beam PET scanner BASTEI installed at GSI. Positioning of a patient is performed with rotation, vertical, and horizontal movements of the couch (1). The individual patient immobilization mask (2) is fixed on the couch. The irradiation beam arrives through the window (3). The PET scanner consists of two heads ( $4 \mathrm{a}$ and $4 \mathrm{~b}$ ).

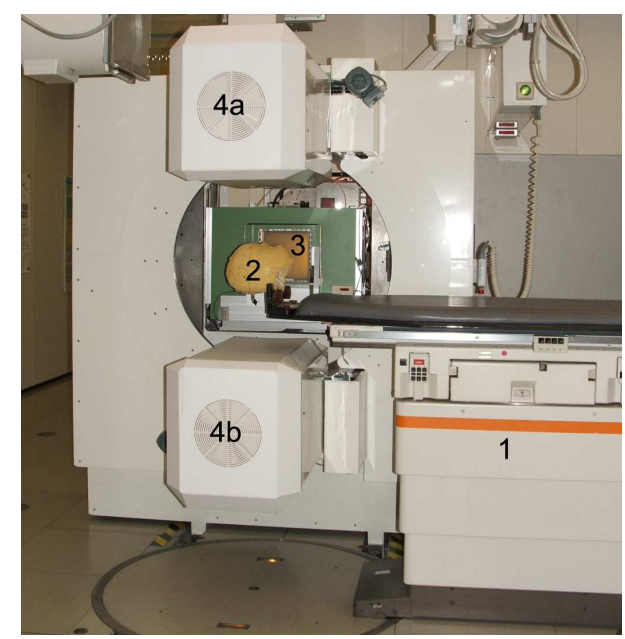


area. In the ideal case $M H=4 M B, M C=0$ and $L C R H=L C R C=100 \%$. In the worst case $M H=M C=M B$ (hot area and cold areas are not recognized from the background) and $L C R H=L C R C=0 \%$.

For in-beam PET any deviations from norm, e.g. changes in the structure (density) of the irradiated tissue or mispositioning of the patient, usually cause a deviation in the maximum particle range. Therefore, the third image quality criterion is based on the light modification of the maximum particle range for real treatment plans and simulation of $\beta^{+}$-activity distributions [3] as shown in (Fig. 3). This quality criterion evaluates the most important imaging characteristics of in-beam PET - the sensitivity of the system to modification of irradiation range. However, it is the most time consuming and requires skills in visual analysis of in-beam PET images.

\subsection{Scheme for quality evaluation}

The integration of an in-beam PET system into particle therapy facility relies on the two main issues: i) choosing of an optimum form of the scanner which fulfills the requirements of the treatment room and provides acceptable imaging capabilities and ii) optimization of reconstruction algorithm for fast delivery of high quality images. The first problem can be solved using a dedicated software tool based on the OpenGL technology. The program receives the dimensions and degrees of freedom for objects (beam nozzle, patient couch, etc.) inside the treatment room as parameters. Then a collision free solution for the integrated in-beam PET scanner is manually generated. The program returns a lookup table with coordinates and position of all detectors of the PET scanner. ML-EM [4], RFS-EM [5], and dTOF [6] reconstruction algorithms can be applied for in-beam PET data. Each reconstruction algorithm has a number of parameters to optimize (number of iterations, size of the image element, etc.). A semiautomatic workflow has been developed in order to find an optimum solution concerning position and geometry of a PET scanner and the reconstruction algorithm (Fig. 4). The steps of the evaluation workflow are performed automatically except the range modification trial which requires experienced human observers. The rough selection of reconstruction parameters and a scanner is

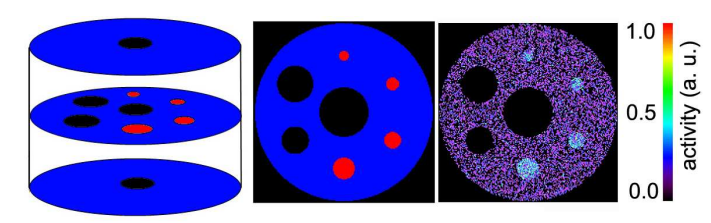

Fig. 2. Left: Schematic view of the NEMA phantom for in-beam PET (up, middle and bottom slices). Central slices of the ideal (center) and simulated (right) NEMA phantoms. The simulated phantom consists of annihilation points distributed according to the predefined concentrations $(4: 1: 0$ for hot, background, and cold areas, respectively). 
done using only two image quality criteria - RMSE and the contrast recovery. Finally, a whole imaging system with the selected parameters must be proven by means of the range modification trial.

\section{Results}

The discussed evaluation workflow and the image quality criteria have been used to find an optimum solution for in-beam PET system. Different reconstruction algorithms (ML-EM [4], RFS-EM [5], and dTOF [6]) have been tested. The dTOF algorithm showed the best image quality. It reconstructed images in real time. However, the detectors required for it are not yet commercially available. More than 50 different schemes for the iterative ML-EM and RFS-EM algorithms have been automatically tested using RMSE and the contrast recovery criteria. The optimum scheme with only 4 iterations through the whole dataset (only $20-120$ s of reconstruction time depending on the image size and the counting statistics) has been found and finally tested with the range modification trial. It was possible to recognize the range modification equivalent to $6 \mathrm{~mm}$ in water for the most typical patient cases. These results allowed to substitue the standard ML-EM algorithm (5-10 min of reconstruction time) with the fast RFS-EM algortihm (20-120 s of reconstruction time) without reducing the sensitivity of the in-beam PET method to the range modifications.

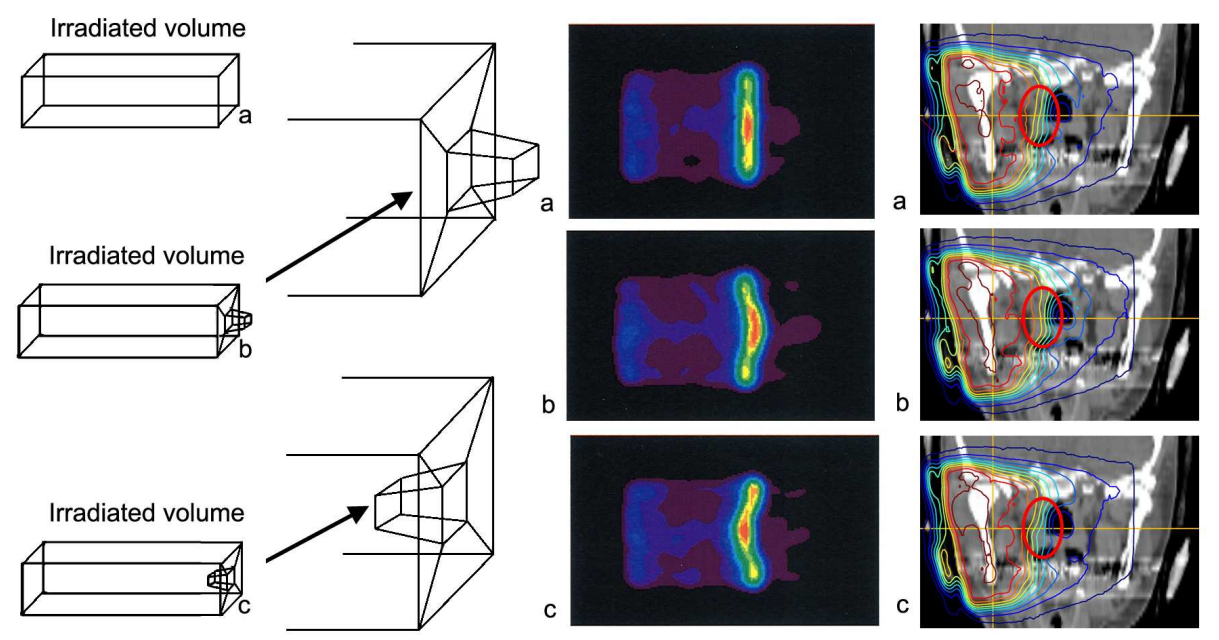

Fig. 3. Schematic view of the range modification (left). Simulations of the $\beta^{+}$-activity distribution in PMMA phantom (centre) and based of the patient plans (right). (a) corresponds to unmodified range, (b) - to enlarged range, and (c) - to reduced range. 


\section{Discussion}

The dedicated image quality criteria for evaluation of an in-beam PET imaging system have been developed. It is suggested to apply the two numeric criteria RMSE and the contrast recovery - first in order to exclude the low image quality cases. Finally, the range modification trial must be performed using data of several patients. This test has to be accomplished by the experienced human observers using representative set of the real patient treatment plans. The sequential way of the image quality evaluation allows to save a lot of time because most of non-optimal parameters can be rejected during the fast automated analysis. The evaluation workflow (Fig. 4) allows to select and evaluate the geometry and a position of the PET scanner in 3D taking into account position and degrees of freedom of other equipment of the treatment room using a dedicated software tool based on the OpenGL technology. Only simulated data are required for this workflow. Therefore, it is possible to perform a fully virtual analysis of imaging capabilities of an in-beam PET system before any prototype is built.

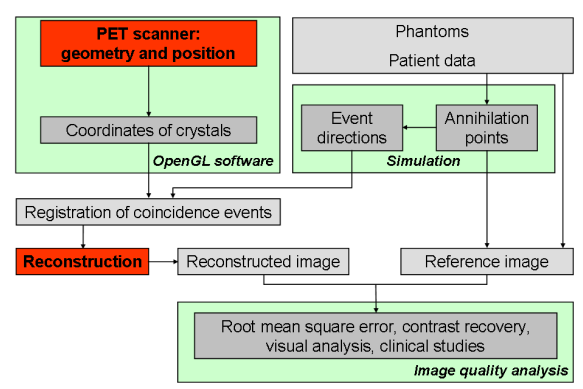

Fig. 4. Evaluation workflow for a PET scanner and reconstruction algorithm. The position and geometery of a PET scanner and a reconstruction algorithm are evaluated using simulated phantoms and patient data. The evaluation is performed using the image quality criteria: RMSE, contrast recovery, visual analysis, and the range modification trial (clinical srudies).

\section{References}

1. Enghardt W, Parodi K, Crespo P, et al. Dose quantification from in-beam positron emission tomography. Radiother Oncol. 2004;73:96-98.

2. Enghardt W, Crespo P, Fiedler F, et al. Charged hadron tumour therapy monitoring by means of PET. Nucl Instrum Methods Phys Res. 2004;525:284-288.

3. Fiedler F, Shakirin G, Crespo P, et al. On the efficiency of ion range determination from in-beam PET data. Radiother Oncol. submitted.

4. Pönisch F, Enghardt W, Lauckner K. Attenuation and scatter correction for inbeam positron emission tomography monitoring of tumour irradiations with heavy ions. Phys Med Biol. 2003;48:2419-2436.

5. Shakirin G, Crespo P, Enghardt W. A method for system matrix construction and processing for reconstruction of in-beam PET data. IEEE Trans Nucl Sci. 2007;54:1710-1716.

6. Crespo P, Shakirin G, Fiedler F, et al. Direct time-of-flight for quantitative, real-time in-beam PET: A concept and feasibility study. phys. med. biol., 52: pp. 6795-6811, 2007. Phys Med Biol. 2007;52:6795-6811. 